Research Article

\title{
Prognostic Significance of Thyroglobulin Antibodies in Differentiated Thyroid Cancer
}

\author{
Jordi L. Reverter (1), ${ }^{1,2}$ Irene Rosas-Allende, ${ }^{3}$ Carlos Puig-Jove, ${ }^{1,2}$ Carles Zafon, ${ }^{2,4}$ \\ Ana Megia, ${ }^{2,5}$ Ignasi Castells, ${ }^{2,6}$ Eduarda Pizarro, ${ }^{2,7}$ Manel Puig-Domingo, ${ }^{1,2}$ \\ and M. Luisa Granada ${ }^{2,3}$ \\ ${ }^{1}$ Departments of Endocrinology and Nutrition, Germans Trias i Pujol Hospital and Research Institute, \\ Universitat Autònoma de Barcelona, CIBERER (ISCIII), Barcelona, Spain \\ ${ }^{2}$ Consortium for the Study of Thyroid Cancer, CECAT, Barcelona, Spain \\ ${ }^{3}$ Clinical Biochemistry, Germans Trias i Pujol Hospital and Research Institute, Universitat Autònoma de Barcelona, \\ CIBERER (ISCIII), Barcelona, Spain \\ ${ }^{4}$ Department of Endocrinology, Hospital Vall d'Hebron, Diabetes and Metabolism Research Unit, \\ Vall d'Hebron Institut de Recerca, Universitat Autònoma de Barcelona, CIBERDEM (ISCIII), Barcelona, Spain \\ ${ }^{5}$ Department of Endocrinology and Nutrition, Hospital Universitari Joan XXIII, Research Institute Pere Virgili, \\ CIBERDEM (ISCIII), Tarragona, Spain \\ ${ }^{6}$ Endocrinology Service, Hospital General de Granollers, Barcelona, Spain \\ ${ }^{7}$ Endocrinology Service, Hospital de Mataró, Barcelona, Spain
}

Correspondence should be addressed to Jordi L. Reverter; reverter.germanstrias@gencat.cat

Received 4 December 2019; Revised 12 February 2020; Accepted 27 February 2020; Published 14 April 2020

Academic Editor: Massimo Tonacchera

Copyright (c) 2020 Jordi L. Reverter et al. This is an open access article distributed under the Creative Commons Attribution License, which permits unrestricted use, distribution, and reproduction in any medium, provided the original work is properly cited.

Objective. To investigate whether variations in thyroglobulin autoantibodies ( $\operatorname{Tg} \mathrm{Ab}$ ) are related to the recurrence or persistence of differentiated thyroid carcinoma (DTC) and may therefore be useful as surrogate tumor markers. Design and Methods. We retrospectively studied 98 subjects (83 women, $47 \pm 15$ years old) from an initial cohort of 1017 patients treated for DTC in five hospitals, with positive $\mathrm{TgAb}$ at any time during the follow-up. Patients presented five different patterns of evolution of serum $\mathrm{TgAb}$ concentrations: (1) stable positive $\mathrm{TgAb},(2)$ de novo appearance, (3) an increase of more than 50\%, (4) $\mathrm{TgAb}$ levels from positive to negative, and (5) a decrease of more than 50\%. Results. In the group of 11 patients with stable TgAb, four cases presented persistence of the disease with structural incomplete response. In the group of 22 patients with sustained increasing trend rising more than $50 \%$ or de novo detectable $\mathrm{TgAb}$ levels, three patients were diagnosed with structural incomplete response. There was no evidence of recurrence or persistence of the disease in any of the 65 patients who showed a significant decrease in $(n=35)$ or disappearance of $(n=30)$ TgAb. Conclusions. Our results suggest that not only the appearance of a significant increase in $\mathrm{TgAb}$ but also stable concentrations of $\mathrm{TgAb}$ should be regarded as a sufficient risk condition for an active search for recurrent or persistent disease. Conversely, a significant decrease in TgAb levels can represent a good prognostic sign.

\section{Introduction}

Differentiated thyroid carcinoma (DTC) has an excellent survival rate. Despite this, recurrences occur in $5 \%-20 \%$ of patients, and because of its prevalence, DTC is the leading cause of death among all endocrine tumors [1]. Serum thyroglobulin $(\mathrm{Tg})$ is produced and secreted exclusively by benign or differentiated malignant thyroid cells, and it is considered a highly sensitive and specific tumor marker for patients with DTC after removal of benign and malignant thyroid tissue by surgery and ${ }^{131}$ I ablation [2]. Thyroglobulin is usually measured by noncompetitive automated immunoassays using two monoclonal antibodies directed against two epitopes of the molecule. Over the years, advances in assay technologies have led to important improvements in the analytical performances of $\mathrm{Tg}$ immunometric assays 
(IMAs), with the sensitivity of detection increasing tenfold from $0.5-1.0 \mu \mathrm{g} / \mathrm{L}$ in the first-generation IMAs to $0.05-0.10 \mu \mathrm{g} / \mathrm{L}$ in the second-generation IMAs. However, the main limitation of determination of $\mathrm{Tg}$ is that it can be modified by serum Tg autoantibodies (TgAb), which give falsely decreased results in IMA assays. Furthermore, this interference is variable among patients and only correlates with $\mathrm{TgAb}$ concentrations [3]. Therefore, the degree of interference cannot be predicted and an accurate Tg value cannot be obtained. It should also be noted that any $\operatorname{TgAb}$ assay will detect only a subset of interfering TgAbs [4].

Serum $\mathrm{TgAb}$ are present in about $60 \%$ of patients with autoimmune thyroid disease and are more frequent in females [5]. It has been reported that approximately $20 \%-25 \%$ of patients are with DTC test positive for TgAb, depending on the specific assay used and the study population [6]. In patients with DTC who test positive for $\mathrm{TgAb}$, an undetectable Tg cannot be judged as the absence of disease and such cases are classified as "indeterminate response" according to the American Thyroid Association (ATA) guidelines [7]. In these patients, it has been suggested that the trend towards increasing $\mathrm{TgAb}$ concentration could reflect changes in thyroid tissue mass [8]. The rationale behind $\operatorname{TgAb}$ measurement and the interpretation of results in DTC patients is based on the theory that the presence of an antigenic stimulus induced by $\mathrm{Tg}$ is what drives $\mathrm{TgAb}$ production [9].

Various authors have suggested that $\mathrm{TgAb}$ can be used as a surrogate tumor marker for disease recurrence during long-term follow-up in DTC [10-13]. However, this is a controversial issue, mainly because the presence of $\mathrm{TgAb}$ may not be useful in a significant proportion of patients due its low prevalence [14], and also because there is as yet no clear definition of the magnitude of $\mathrm{TgAb}$ level variation and the period of time over which it occurs that would be necessary for it to be regarded as a significant trend in their concentrations.

The aim of this study was to investigate the prevalence of $\mathrm{TgAb}$ in a large cohort of DTC patients and its potential usefulness as a surrogate tumor marker by evaluating whether variations in $\operatorname{TgAb}$ levels are associated with disease activity.

\section{Materials and Methods}

2.1. Patients. We retrospectively studied patients treated for DTC between 2010 and 2016 at the Endocrinology Departments of five hospitals in the region of Catalonia, Spain: the Germans Trias i Pujol University Hospital in Badalona, the Vall d'Hebron University Hospital in Barcelona, the Joan XXIII Hospital in Tarragona, the Granollers General Hospital in Granollers, and the Mataró Hospital in Mataró. Patients were treated with complete thyroidectomy, additional lymph node dissection if indicated, suppressive levothyroxine treatment, and postoperative remnant ablation with ${ }^{131}$ I according to ATA guidelines [7]. Laboratory evaluation consisted of taking measurements of serum $\mathrm{Tg}$, $\mathrm{TgAb}$, and TSH at least once every year. Recurrence was defined as detection of structural disease by ultrasound or other image explorations, whole-body scan, or presence of malignant cells in a fine-needle aspiration biopsy or in tissue obtained during reoperation. Patient characteristics (gender and age), laboratory measurements (TSH, Tg, and $\mathrm{TgAb}$ levels), tumor characteristics (histological subtype: papillary or follicular), and data regarding disease status during follow-up and duration of follow-up were also recorded. All patients with at least one year of follow-up and at least two determinations of $\mathrm{Tg}$ and $\mathrm{TgAb}$, measured in a period of at least 6 months, were included in this study. Patients with TgAb levels below functional sensitivity of the assay in all the samples obtained during the follow-up were excluded.

The study was conducted in accordance with the Declaration of Helsinki and was approved by the local Human Research Ethics Committee of the Hospital Germans Trias i Pujol. Consent was obtained from each patient after full explanation of the purpose and nature of all procedures used.

2.2. Laboratory Methods. Thyroglobulin determinations were carried out by Elecsys ${ }^{\circledR}$ electro chemiluminescent electromagnetic immunoassay (ICMA) (Roche Diagnostics). $\operatorname{TgAb}$ were measured by chemiluminescent immunoassay using either the Liaison ${ }^{\circledR}$ system (DiaSorin, Saluggia, Italy) in one of the participating centers and the Immulite $^{\circledR} 2000$ XPi analyzer (Siemens Healthcare Diagnostics, Llanberis, UK) in the others. Functional sensitivity (FS), defined as the lowest $\operatorname{TgAb}$ concentration that can be measured with an interassay coefficient of variation $<20 \%$, was $10 \mathrm{UI} / \mathrm{mL}$. A result was considered positive for $\mathrm{TgAb}$ if serum levels were $\geq 10 \mathrm{IU} / \mathrm{mL}$ (above FS) in samples measured by the Liaison ${ }^{\circledR}$ and $\geq 20 \mathrm{IU} / \mathrm{mL}$ in those measured with the Immulite ${ }^{\circledR} 2000$ XPi.

Given that $\operatorname{TgAb}$ may rise transiently after surgery and ${ }^{131}$ I ablation therapy, TgAb were measured at least 6 months after these treatment procedures.

Based on $\mathrm{TgAb}$ presence and changes in serum $\mathrm{TgAb}$ concentrations, patients presented one of the five different patterns: (1) stable positive TgAb concentrations with variations less than 50\% throughout follow-up, (2) increased levels of $\operatorname{TgAb}$ above functional sensitivity (de novo appearance from negative to positive), (3) sustained increasing trend (increasing more than 50\% in levels), (4) TgAb levels decreasing below FS with a shift in $\mathrm{TgAb}$ status from positive to persistently negative, or (5) levels of $\mathrm{TgAb}$ decreasing more than $50 \%$.

2.3. Imaging Modalities. Neck ultrasound was performed at least yearly, and further imaging techniques $\left({ }^{131}\right.$ Iodine, CT scan, MRI, 18-FDG PET/CT) were used if indicated.

2.4. Recurrent or Persistent Disease. Recurrence or persistence of the disease had to be proven by cytology, histology, or positive post- ${ }^{131} \mathrm{I}$ therapy scan. In this study, the ATA management guidelines criteria for recurrence and persistence of the disease were used [7].

2.5. Statistical Analysis. Descriptive statistics were applied to all collected variables expressed as frequencies for categorical data or mean values \pm standard deviations for 
continuous data. Group comparisons were carried out using the chi-square test. A $p$ value less than 0.05 was considered significant. Statistical analysis was performed using the IBM SPSS ${ }^{\circledR}$ Statistics 24 software package (IBM Corp., Armonk, NY, USA).

\section{Results}

3.1. Patients. From an initial cohort of 1017 patients with DTC, 98 ( $n=83$ females, $47 \pm 15 \mathrm{y}$ ), representing 9.5\%, presented positive $\mathrm{TgAb}$ at some point during follow-up. Table 1 shows the characteristics of these patients. This group included papillary thyroid cancer $(n=86)$ and follicular thyroid carcinoma $(n=12)$. Patient's initial Tg levels were $0.75 \pm 1.3 \mathrm{ng} / \mathrm{mL}$. In terms of $\mathrm{TgAb}$ status, 11 patients had stable positive TgAb concentrations, 16 patients had de novo appearance, and 6 patients had a significant increase in TgAb. On the other hand, in 65 patients, TgAb levels decreased above FS $(n=35)$ or $>50 \%(n=30)$ during follow-up. Figure 1 represents a flowchart of the results.

3.2. Relationship between $\mathrm{TgAb}$ and Recurrence or Persistence of the Disease. Table 2 shows the characteristics of the 7 patients with disease recurrence according to $\mathrm{TgAb}$ levels during the follow-up. In the group of 11 patients with stable $\mathrm{TgAb}$, the mean follow-up period was $78.9 \pm 9.0$ months, and four cases presented persistence of the disease with structural incomplete response. In only one of the 6 patients with sustained increasing trend rising more than $50 \%$ was recurrence detected. Sixteen patients presented de novo detectable $\mathrm{TgAb}$ levels and two of these patients were diagnosed with structural incomplete response. The mean follow-up period in the group of increasing or de novo appearance $\mathrm{TgAb}$ was $75.7 \pm 10.1$ months.

There was no evidence of recurrence or persistence of the disease in any of the 65 patients who showed a significant decrease in $(n=35)$ or disappearance of $(n=30)$ TgAb after a mean follow-up of $65 \pm 10.9$ months.

When patients with pattern type 1 (increase) were compared with those in pattern type 2 (decrease), no significant differences were observed regarding persistence or recurrence of the disease $(p<0.2)$. The relative risk of having active disease was higher but not significantly different in patients with stable $\mathrm{TgAb}$ with respect to those with de novo appearance $(p=0.3)$ or a significant increase $(p=0.2)$. Patients with positive TgAb presented a highly significant $R R$ of persistent or recurrent disease (not quantifiable due to the absence of cases with active disease in the group of patients whose $\mathrm{TgAb}$ decreased significantly or disappeared).

\section{Discussion}

First, in this study involving a relatively large cohort of patients with DTC and positive $\mathrm{TgAb}$ at some point during follow-up, the prevalence of positive $\mathrm{TgAb}$ was relatively low, less than $10 \%$, with respect to previous reports [14-16]. Second, a decrease in $\mathrm{TgAb}$ to undetectable levels below the FS of the method or a significant decrease $>50 \%$ identified those cases with no evidence of disease recurrence or
TABle 1: Patient demographics and characteristics.

\begin{tabular}{lc}
\hline Sex $(\mathrm{M} / \mathrm{F})$ & $15 / 83$ \\
Mean age, years (SD) & $47( \pm 15)$ \\
Family history neoplasia, $n$ & 39 \\
Family history thyroid disease, $n$ & 29 \\
Prior neck irradiation, $n$ & 8 \\
Histologic subtype, $n$ & \\
Classical papillary & 80 \\
Tall cell & 1 \\
Sclerosing & 4 \\
Hürthle & 1 \\
Follicular & 12 \\
Mean size, cm (SD) & $2.3( \pm 1.7)$ \\
Multifocality, $n$ & 33 \\
Extrathyroidal extension, $n$ & 23 \\
Lymphovascular invasion & 17 \\
\hline TNM classification, $n$ & \\
T1 & 38 \\
T2 & 22 \\
T3 & 28 \\
T4 & 10 \\
N1 & 48 \\
M1 & 10 \\
\hline Stage, $n$ & \\
Stage I & \\
Stage II & \\
Stage III & 26 \\
Stage IVA & 18 \\
\hline
\end{tabular}

persistence; this group was strongly represented as it corresponded to 65 out of the 98 patients. And finally, the cases with active disease were detected in patients with stable levels of a significant increase in or de novo appearance of $\mathrm{TgAb}$, thus supporting the usefulness of the measurement of $\mathrm{TgAb}$ as a surrogate marker of active disease in DTC [17].

From a clinical point of view, these results suggest that only a minority of cases of DTC show positive TgAb during follow-up. This low prevalence of $\operatorname{TgAb}$, together with the fact that in more than half of cases, $\operatorname{TgAb}$ disappear or decrease during follow-up, raises the question of whether its measurement would be of major utility in daily clinical practice. The prevalence reported in various studies on this topic is not only higher $(25 \%)$ than in the general population (10\%) but also higher than that seen in our results $[4,16]$. Based on our data, the actual impact on DTC populations may be limited. As the prevalence of positive $\mathrm{TgAb}$ in our DTC population was $10 \%$ and the probability of recurrence of disease in papillary thyroid carcinoma (PTC) has been reported to reach $20 \%$ at some point during the patient's lifetime, the combined probability of recurrence in patients with positive $\mathrm{TgAb}$ is 2 out of every 100 DTC patients. In connection with this, two long-term retrospective studies, covering follow-up periods of 12 to 29 years, detected only 2.5 patients per year with positive $\operatorname{TgAb}[11,18]$. Population factors may be at the root of this difference in $\operatorname{TgAb}$ prevalence [19].

Independent of its prevalence, in those patients showing positive $\operatorname{Tg} \mathrm{Ab}$, who are considered a diagnostic challenge, serum levels of $\operatorname{TgAb}$ have been proposed as a surrogate 


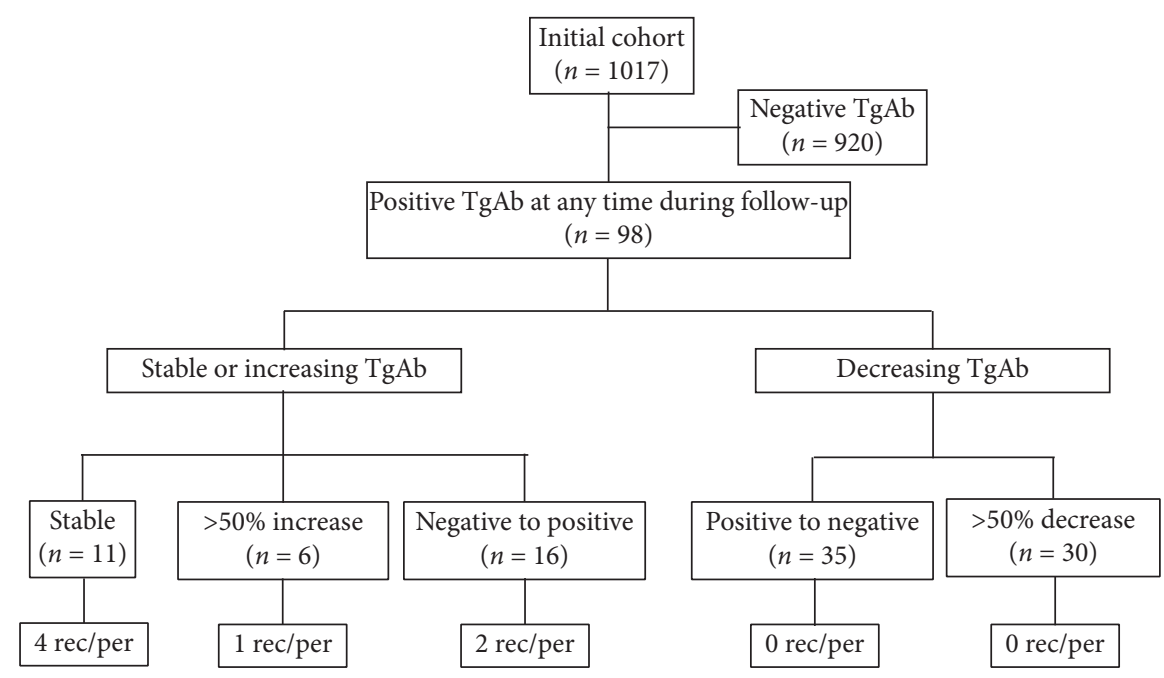

$\mathrm{TgAb}$ : thyroglobulin auto antibodies

rec/per: thyroid carcinoma recurrence or persistence

FIgURE 1: Flow chart of the study population and results.

TABLE 2: Characteristics of the patients with disease recurrence according to thyroglobulin autoantibodies levels during the follow-up.

\begin{tabular}{|c|c|c|c|c|c|c|c|}
\hline \multirow{2}{*}{$\begin{array}{l}\operatorname{TgAb} \\
\operatorname{Sex}(M / F)\end{array}$} & \multicolumn{4}{|c|}{ Stable } & \multirow{2}{*}{$\frac{>50 \% \text { increase }}{\mathrm{F}}$} & \multicolumn{2}{|c|}{ de novo appearance } \\
\hline & M & $\mathrm{F}$ & $\mathrm{F}$ & $\mathrm{F}$ & & $\mathrm{F}$ & $\mathrm{F}$ \\
\hline Age (years) & 50 & 73 & 38 & 52 & 77 & 70 & 75 \\
\hline Histology & Papillary & Papillary & Papillary & Papillary & Papillary & Papillary & Papillary \\
\hline Initial TNM & T3N1M0 & T4N1M0 & T2N1M0 & T4N1M1 & T4N1M0 & T4N1M0 & T3N1M0 \\
\hline Diagnostic procedure & $\mathrm{PET} / \mathrm{TC}$ & $\mathrm{PET} / \mathrm{TC}$ & Ultrasound & $\mathrm{PET} / \mathrm{TC}$ & $\mathrm{PET} / \mathrm{TC}$ & Ultrasound & Ultrasound \\
\hline Recurrence location & Lung M1 & Lung M1 & Neck N1 & Lung M1 & Lung M1 & Neck N1 & Neck N1 \\
\hline Recurrence time (months) & 12 & 14 & 7 & 23 & 36 & 48 & 23 \\
\hline \multicolumn{8}{|l|}{$\mathrm{Tg}(\mathrm{ng} / \mathrm{ml})$} \\
\hline Initial & 0.2 & 5.8 & 0.4 & 0.2 & 0.2 & 176 & 0.2 \\
\hline Recurrence & 0.1 & 0.6 & 0.1 & 0.2 & 0.2 & 19 & 0.3 \\
\hline \multicolumn{8}{|l|}{$\mathrm{TgAb}(\mathrm{IU} / \mathrm{ml})$} \\
\hline Initial & 128 & 3000 & 81 & 900 & 221 & Negative & Negative \\
\hline Recurrence & 83 & 2500 & 71 & 700 & 2500 & 63 & 172 \\
\hline
\end{tabular}

PET/TC: 18F-fluorodeoxyglucose positron emission tomography/computed tomography; Tg: thyroglobulin; TgAb: thyroglobulin autoantibodies; N1: lymph node metastasis; M1: distant metastasis.

tumor marker $[10,11,15,16]$. Our results are in accordance with the ATA guidelines statement [7], which indicates that a decline in $\operatorname{TgAb}$ levels over time is considered a good prognostic sign [20], while rising antibody levels, in the absence of an acute injury to the thyroid, significantly increase the risk of persistent or recurrent thyroid cancer [21]. Specifically, recurrent or progressive disease should be suspected in those patients initially positive for $\operatorname{TgAb}$ that then become negative but subsequently show rising levels of $\operatorname{TgAb}$ [7]. In our study, all patients with persistent or recurrent disease had de novo appearance of $\mathrm{TgAb}$ or a significant $>50 \%$ increase during follow-up, but also no active DTC was observed in any patient in whom $\operatorname{TgAb}$ levels became undetectable or decreased significantly over time. These results agree with a previous prospective study of 576 patients with DTC, which found no statistically significant difference in recurrence development between patients with persistent undetectable $\operatorname{TgAb}(2.6 \%)$ compared with those with borderline $\operatorname{TgAb}$ (3.2\%) during follow-up. However, patients with borderline $\mathrm{TgAb}$ were more likely to progress to elevated $\operatorname{TgAb}$, and this event was significantly associated with recurrence [22].

The interference of $\operatorname{TgAb}$ in $\mathrm{Tg}$ determinations is an issue that has not yet been resolved in the clinical assessment of thyroid carcinoma patients classified as "undetermined response." In the absence of alternative methods, neck ultrasound and other image explorations have become the basis of risk stratification [7]. Some studies have proposed that $\mathrm{TgAb}$ persistence suggests persistence or recurrence of the disease $[20,21]$ even when limiting the time frame to the first year after treatment in TgAb positive PTC patients [23]. Our study does not support these findings. In our cohort, the pattern depicting the persistence of stable levels or increase in $\operatorname{Tg} \mathrm{Ab}$ throughout follow-up is associated with active disease. However, other studies have found no correlation between persistent $\mathrm{TgAb}$ and higher frequency of recurrence or mortality [24]. 
An important point to consider is the need to establish clear definitions for trends in $\operatorname{TgAb}$ levels and the level at which the $\operatorname{TgAb}$ is considered as positive. In this regard, reports of the cutoff point of serum $\mathrm{TgAb}$ levels are scarce and inconsistent [25]. Some authors have used the reference range provided by the manufacturer as a limit, but this specific value was established to identify autoimmune thyroid disease and not to define interference with $\mathrm{Tg}$ [16]. We consider, according to other authors [11], that TgAb levels "positive" or "negative" depend on the functional sensitivity of the assay since interference can occur at detectable $\mathrm{TgAb}$ concentrations below the reference limit (borderline $\mathrm{TgAb}$ ). In addition, the quantification of $\operatorname{TgAb}$ levels allows the evaluation of variations over time, which is also very important for defining the trend pattern of an individual patient [8]. Some of the reasons for the discrepancies between studies may be related to some selection bias or differences in study design.

Another potential source of discrepancy is the two different methods used to analyze $\mathrm{TgAb}$ in our study. In this sense, despite being standardized according to the same World Health Organization International Standard 65/93, these two tests give different numerical results. To validate our results, we made a previous comparison of the results obtained by these two trials in a sample of 80 subjects, showing that they had a high level of correlation $(r=0.92$, $p<0.001$ ) with proportional differences (slope $=0.70$ ), which indicates that the results obtained by Immulite (Siemens) were consistently higher than those obtained with Liason (DiaSorin) (data not shown).

The presence of endogenous $\mathrm{TgAb}$ may interfere in $\mathrm{Tg}$ measurements, resulting in falsely high (in competitive radioimmunoassays) or falsely low (with current immunometric assays) results. The cutoff level is used to determine the qualitative status of $\operatorname{TgAb}$ (positive or negative), and therefore, the interference of the $\mathrm{Tg}$ assays has been established as the functional sensitivity of the tests based on the ratio $\mathrm{Tg}$-IMA to $\mathrm{Tg}$-RIA. It has been shown that when the manufacturer's recommended cutoff point (used to detect thyroid autoimmunity) was used to determine the presence of $\operatorname{TgAb}$, some samples showing interference were falsely classified as negative for $\operatorname{TgAb}$ and this lowered the limit of detection of $\mathrm{TgAb}$. Functional sensitivity minimizes false negative ratings. For this reason, it is preferred to use functional sensitivity to assess the qualitative state of $\mathrm{TgAb}$.

Persistent $\operatorname{TgAb}$ in patients without evidence of disease may be explained by different pathogenic mechanisms [20]. First, small amounts of residual normal thyroid tissue could be present. Second, the presence of $\operatorname{TgAb}$ could be associated with the coexistence of underlying autoimmune thyroiditis. And finally, $\operatorname{TgAb}$ production may be carried out by some lymphocytic memory cells [19]. The immune response may be triggered as a reaction to the inflammatory process associated with the tumorigenesis, in which $\mathrm{Tg}$ molecules modified by posttranslational changes behave like antigens with great immunogenicity.

Concentrations of $\mathrm{TgAb}$ are currently measured with immunoassays using monoclonal antibodies, and their results are traceable to the IRP 65/93 reference standard. In spite of this, the limits of quantification of the different assays and the discriminant values that each manufacturer recommends considering that a sample contains antibodies against $\mathrm{Tg}$ can vary by a factor of up to 200 . The heterogeneity of the epitopes to which the antibodies are directed and their recognition in different $\operatorname{TgAb}$ assays is a known cause of variation [26]. In this regard, patients with DTC with restriction of the $\operatorname{TgAb}$ epitope similar to Hashimoto's disease have a higher rate of disease recurrence or persistence than in patients with nonspecific reactivity, which suggests that $\operatorname{TgAb}$ epitope specificities may have prognostic value [27].

The main strengths of our study are the strict follow-up during an average period of four years, the precise analytical determinations, and the homogeneity of the sample of DTC patients coming from the same geographical area of Northeastern Spain. The data presented in this study are not new in themselves but, in our opinion, they broaden the base of scientific knowledge on a controversial topic of clinical significance. In addition, thyroid autoimmunity has a multifactorial etiology with a complex interaction of environmental factors in genetically susceptible individuals. Therefore, the influence of thyroid autoimmunity in patients with thyroid cancer could be significantly different depending on the populations studied, and providing data in our area is a contribution that we consider valuable. Among the weaknesses is the retrospective design.

In conclusion, from a clinical perspective, our results show a low prevalence of $\operatorname{TgAb}$ in CDT patients. When $\operatorname{Tg} \mathrm{Ab}$ is detected in a given patient, temporally close follow-up is mandatory for the definition of any $\mathrm{TgAb}$ temporal variation pattern, thus suggesting that not only the appearance of $\operatorname{TgAb}$ or significant increase in $\operatorname{TgAb}$ but also stable $\operatorname{TgAb}$ concentrations should be considered a warning sign. In these cases, active search for recurrent or persistent disease by means of highly sensitive image scans is warranted. By contrast, a significant decline in or stable persistence of $\mathrm{TgAb}$ levels can be considered a good prognostic sign.

\section{Data Availability}

The clinical and analytical data used to support the findings of this study are available upon request to the corresponding author.

\section{Conflicts of Interest}

The authors declare that there are no conflicts of interest.

\section{References}

[1] H. Lim, S. S. Devesa, J. A. Sosa, D. Check, and C. M. Kitahara, "Trends in thyroid cancer incidence and mortality in the United States, 1974-2013," JAMA, vol. 317, no. 13, pp. 1338-1348, 2017.

[2] M. Hocevar, M. Auersperg, and L. Stanovnik, "The dynamics of serum thyroglobulin elimination from the body after thyroid surgery," European Journal of Surgical Oncology (EJSO), vol. 23, no. 3, pp. 208-210, 1997. 
[3] B. C. Netzel, S. K. G. Grebe, B. G. Carranza Leon et al., "Thyroglobulin $(\mathrm{Tg})$ testing revisited: $\mathrm{Tg}$ assays, $\mathrm{Tg} \mathrm{Ab}$ assays, and correlation of results with clinical outcomes," The Journal of Clinical Endocrinology \& Metabolism, vol. 100, no. 8, pp. E1074-E1083, 2015.

[4] M. Schlumberger, A. Hitzel, M. E. Toubert et al., "Comparison of seven serum thyroglobulin assays in the follow-up of papillary and follicular thyroid cancer patients," The Journal of Clinical Endocrinology \& Metabolism, vol. 92, no. 7, pp. 2487-2495, 2007.

[5] J. G. Bonichon, N. W. Staehling, W. D. Flanders et al., "Serum TSH, T4, and thyroid antibodies in the United States population (1988 to 1994): national health and nutrition examination survey (NHANES III)," The Journal of Clinical Endocrinology \& Metabolism, vol. 87, no. 2, pp. 489-499, 2002.

[6] F. A. Verburg, M. Luster, C. Cupini et al., "Implications of thyroglobulin antibody positivity in patients with differentiated thyroid cancer: a clinical position statement," Thyroid, vol. 23, no. 10, pp. 1211-1225, 2013.

[7] B. R. Haugen, E. K. Alexander, K. C. Bible et al., “2015 American thyroid association management guidelines for adult patients with thyroid nodules and differentiated thyroid cancer: the American thyroid association guidelines task force on thyroid nodules and differentiated thyroid cancer," Thyroid, vol. 26, no. 1, pp. 1-133, 2016.

[8] C. A. Schuff, M. Takeuchi, M. Kazarosyan et al., "Serum thyroglobulin autoantibodies: prevalence, influence on serum thyroglobulin measurement, and prognostic significance in patients with differentiated thyroid Carcinoma1," The Journal of Clinical Endocrinology \& Metabolism, vol. 83, no. 4, pp. 1121-1127, 1998.

[9] L. Chiovato, F. Latrofa, L. E. Braverman et al., "Disappearance of humoral thyroid autoimmunity after complete removal of thyroid antigens," Annals of Internal Medicine, vol. 139, no. 5 Part_1, pp. 346-351, 2003.

[10] C.-J. Hsieh and P.-W. Wang, "Sequential changes of serum antithyroglobulin antibody levels are a good predictor of disease activity in thyroglobulin-negative patients with papillary thyroid carcinoma," Thyroid, vol. 24, no. 3, pp. 488-493, 2014.

[11] S. G. A. de Meer, W. M. C. M. Vorselaars, J. W. Kist et al., "Follow-up of patients with thyroglobulin-antibodies: rising $\mathrm{Tg}-\mathrm{Ab}$ trend is a risk factor for recurrence of differentiated thyroid cancer," Endocrine Research, vol. 42, no. 4, pp. 302310, 2017.

[12] R. Görges, M. Maniecki, W. Jentzen et al., "Development and clinical impact of thyroglobulin antibodies in patients with differentiated thyroid carcinoma during the first 3 years after thyroidectomy," European Journal of Endocrinology, vol. 153, pp. 49-55, 2005.

[13] J. H. Seo, S. W. Lee, B.-C. Ahn, and J. Lee, "Recurrence detection in differentiated thyroid cancer patients with elevated serum level of antithyroglobulin antibody: special emphasis on using18F-FDG PET/CT," Clinical Endocrinology, vol. 72, no. 4, pp. 558-563, 2010.

[14] C. Spencer and S. Fatemi, "Thyroglobulin antibody (TgAb) methods-strengths, pitfalls and clinical utility for monitoring TgAb-positive patients with differentiated thyroid cancer," Best Practice \& Research Clinical Endocrinology \& Metabolism, vol. 27, no. 5, pp. 701-712, 2013.

[15] F. Pacini, S. Mariotti, N. Formica et al., "Incidence of antithyroid autoantibodies in thyroid cancer patients," Thyroid Autoimmunity, vol. 119, pp. 571-573, 1987.
[16] P. W. S. Rosário, F. F. R. Maia, T. A. Fagundes, F. P. Vasconcelos, L. D. Cardoso, and S. Purisch, "Antithyroglobulin antibodies in patients with differentiated thyroid carcinoma: methods of detection, interference with serum thyroglobulin measurement and clinical significance," Arquivos Brasileiros de Endocrinologia \& Metabologia, vol. 48, no. 4, pp. 487-492, 2004.

[17] D. Rubello, D. Casara, M. E. Girelli, M. Piccolo, and B. Busnardo, "Clinical meaning of circulating antithyroglobulin antibodies in differentiated thyroid cancer: a prospective study," Journal of Nuclear Medicine, vol. 33, pp. 1478-1480, 1992.

[18] S.-F. Kuo, T.-C. Chao, H.-Y. Chang et al., "Prognosis of papillary thyroid cancers with positive serum thyroglobulin antibody after total thyroidectomy," Asian Journal of Surgery, vol. 40, no. 3, pp. 186-192, 2017.

[19] Y. Nagayama, "Thyroid autoimmunity and thyroid cancer the pathogenic connection: a 2018 update," Hormone and Metabolic Research, vol. 50, no. 12, pp. 922-931, 2018.

[20] W. G. Kim, J. H. Yoon, W. B. Kim et al., "Change of serum antithyroglobulin antibody levels is useful for prediction of clinical recurrence in thyroglobulin-negative patients with differentiated thyroid carcinoma," The Journal of Clinical Endocrinology \& Metabolism, vol. 93, no. 12, pp. 4683-4689, 2008.

[21] J.-K. Chung, Y. J. Park, T. Y. Kim et al., "Clinical significance of elevated level of serum antithyroglobulin antibody in patients with differentiated thyroid cancer after thyroid ablation," Clinical Endocrinology, vol. 57, no. 2, pp. 215-221, 2002.

[22] M. C. S. Côrtes, P. W. Rosario, L. F. F. Oliveira, and M. R. Calsolari, "Clinical Impact of Detectable antithyroglobulin antibodies below the reference Limit (borderline) in patients with papillary thyroid carcinoma with undetectable serum thyroglobulin and normal neck ultrasonography after ablation: a prospective study," Thyroid, vol. 28, no. 2, pp. 229-235, 2018.

[23] C. Durante, S. Tognini, T. Montesano et al., "Clinical aggressiveness and long-term outcome in patients with papillary thyroid cancer and circulating anti-thyroglobulin autoantibodies," Thyroid, vol. 24, no. 7, pp. 1139-1145, 2014.

[24] S. Bruno, K. Ro, O. Levin, P. H. G. Ituarte, A. Harari, and M. W. Yeh, "Thyroglobulin antibody levels do not predict disease status in papillary thyroid cancer," Clinical Endocrinology, vol. 81, no. 2, pp. 271-275, 2014.

[25] C. Spencer, I. Petrovic, and S. Fatemi, "Current thyroglobulin autoantibody $(\mathrm{Tg} \mathrm{Ab})$ assays often fail to detect interfering $\mathrm{TgAb}$ that can result in the reporting of falsely low/undetectable serum Tg IMA values for patients with differentiated thyroid cancer," The Journal of Clinical Endocrinology \& Metabolism, vol. 96, no. 5, pp. 1283-1291, 2011.

[26] O. E. Okosieme, C. Evans, L. Moss, A. B. Parkes, L. K. E. Premawardhana, and J. H. Lazarus, "Thyroglobulin antibodies in serum of patients with differentiated thyroid cancer: relationship between epitope specificities and thyroglobulin recovery," Clinical Chemistry, vol. 51, no. 4, pp. 729-734, 2005.

[27] G. A. Lupoli, O. E. Okosieme, C. Evans et al., "Prognostic significance of thyroglobulin antibody epitopes in differentiated thyroid cancer," Journal of Clinical Endocrinology and Metabolism, vol. 100, no. 1, pp. 100-108, 2015. 